\section{Immunization update 2005: Stepping forward}

$\mathrm{T}$ his year marks a major step forward in improving access for all children and youth in Canada to the routine vaccines recommended by the National Advisory Committee on Immunization (Figure 1) (1). The disparity in vaccine access between have and have not regions noted previously $(2,3)$ has been narrowed through the collaboration of the federal and provincial/territorial governments in the area of immunization (4). Unfortunately, harmonization of schedules across the country still remains a dream (Table 1). This lack of harmony continues to put children and youth who relocate at potential risk for missing out on a routine vaccine due to schedule timing differences. This is not a new problem (5) but one that is now more exaggerated due to the added variation in schedules and catch-up programs with the 'newer' vaccines (eg, varicella, conjugated pneumococcal and conjugated meningococcal vaccines) (Tables 1 and 2). Determining what a child or youth needs on arrival into a new region is often not an easy task. By referring to Tables 1 and 2, doctors and nurses will be better able to plan an immunization transition program for the children and youth who have changed jurisdictions. These tables are regularly updated with the help of the Canadian Nurses Coalition on Immunization and are available at the Canadian Paediatric Society's Web sites (<www.cps.ca> and <www.caringforkids.ca>), with a note of the posting date marking the most recent update. The Public Health Agency of Canada's Web site (<www.phac-aspc.gc.ca/im/ptimprog-progimpt/index.html $>$ ) is a good source for regularly updated tables. Please note that because changes do occur often, it may be wise to also check the individual provincial and territorial Web sites for the most up-to-date information.

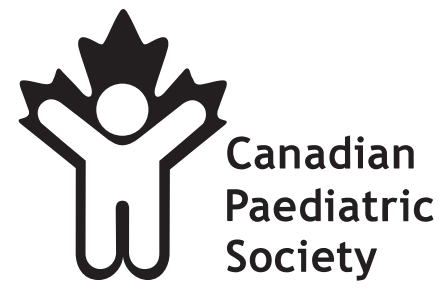

\begin{tabular}{|c|c|c|c|c|c|c|c|c|c|c|}
\hline Vaccine & $2 m$ & $4 \mathrm{~m}$ & $6 \mathrm{~m}$ & $12 \mathrm{~m}$ & $15 \mathrm{~m}$ & $18 \mathrm{~m}$ & $23 \mathrm{~m}$ & $4-6 y$ & $9-12 y$ & $13-16 y$ \\
\hline DTaP & 1 & 2 & 3 & & & 4 & & 5 & & \\
\hline IPV & 1 & 2 & [3] & & & 4 & & 5 & & \\
\hline Hib & 1 & 2 & 3 & & & 4 & & & & \\
\hline dTap & & & & & & & & & & 1 \\
\hline PC & 1 & 2 & 3 & & 4 & & & & & \\
\hline MC & 1 & 2 & 3 & $\begin{array}{l}\text { ALT: } \\
1 \text { dose }\end{array}$ & & & $\begin{array}{l}\text { Catch-up } \\
1 \text { dose }\end{array}$ & & & \\
\hline HBV & & $\begin{array}{l}\text { Infant: } \\
\times 3 \text { dose }\end{array}$ & & & & OR & & & $\begin{array}{l}\text { ALT: } A \\
\times 3 d\end{array}$ & $\begin{array}{l}\text { dolescent } \\
\text { ses }\end{array}$ \\
\hline MMR & & & & 1 & & 2 & OR & 2 & & \\
\hline v & & & & & 1 & & & $\begin{array}{l}\text { Catch-up } \\
1 \text { dose }\end{array}$ & & $\begin{array}{l}\text { Catch-up } \\
2 \text { doses }\end{array}$ \\
\hline FLU & & & \multicolumn{5}{|c|}{ Yearly dose (2 doses in first year) } & \multicolumn{3}{|c|}{$\begin{array}{l}\text { Yearly dose, but } 2 \text { doses in } \\
\text { first year until } 9 \text { years old }\end{array}$} \\
\hline
\end{tabular}

Figure 1) Adapted from the National Advisory Committee on Immunization's routine childhood immunization schedule by Dr Ben Tan, Department of Paediatrics, University of Saskatchewan, Saskatoon, Saskatchewan. Numbers in parentheses indicate doses that are not needed routinely, but can be included for convenience. Within the same row, the bolder colour indicates the 'recommended' time, while the lighter hue indicates when immunization may still be considered. ALT Alternate recommended age; Catch-up Immunize if not previously vaccinated; DaPT Diphtheria-acellular pertussis-tetanus; dTap Tetanus-diphtheria acellular pertussis vaccine; FLU Influenza; HBV Hepatitis B virus; Hib Haemophilus influenzae type $b$ vaccine; IPV Inactivated polio vaccine; MC Meningococcal conjugate; MMR Measles-mumps-rubella vaccine; $m$ Months; PC Pneumococcal conjugate; V Varicella; y Years

TABLE 1

Routine immunization programs for infants and children in Canada, June 2005

\begin{tabular}{|c|c|c|c|c|c|c|c|c|c|}
\hline $\begin{array}{l}\text { Province or } \\
\text { Territory }\end{array}$ & $\begin{array}{l}\text { T/IPV/Hib } \\
6,18 \mathrm{mo})\end{array}$ & Hepatitis B & MMR (2 doses) & $\begin{array}{r}\text { DaPT/IPV } \\
\text { (4-6 yrs) }\end{array}$ & dTap & Varicella & $\begin{array}{l}\text { Meningococcal } \\
\text { conjugate }\end{array}$ & $\begin{array}{l}\text { Pneumococcal } \\
\text { conjugate }\end{array}$ & Influenza \\
\hline British Columbia & $\checkmark$ & $2,4,6 \mathrm{mo}$ & $12,18 \mathrm{mo}$ & $\checkmark$ & Grade 9 & $12 \mathrm{mo}$ & $2,12 \mathrm{mo}$ & $2,4,6,18 \mathrm{mo}$ & $6-23 \mathrm{mo}$ \\
\hline Alberta & $\checkmark$ & Grade 5 & 12 mo, 4-6 yrs & $\checkmark$ & Grade 9 & $12 \mathrm{mo}$ & $2,4,6 \mathrm{mo}$ & $2,4,6,18 \mathrm{mo}$ & $6-23 \mathrm{mo}$ \\
\hline Saskatchewan & $\checkmark$ & Grade 6 & $12,18 \mathrm{mo}$ & $\checkmark$ & Grade 8 & $12 \mathrm{mo}$ & $12 \mathrm{mo}$ & $2,4,6,18 \mathrm{mo}$ & N/A \\
\hline Manitoba & $\checkmark$ & Grade 4 & 12 mo, 4-6 yrs & $\checkmark$ & Grade 9 & $12 \mathrm{mo}$ & Grade 4 & $2,4,6,18 \mathrm{mo}$ & $6-23 \mathrm{mo}$ \\
\hline Ontario & $\checkmark$ & Grade 7 (2 doses) & 12 mo, 4-6 yrs & $\checkmark$ & $14-16$ yrs & $15 \mathrm{mo}$ & $12 \mathrm{mo}$ & $2,4,6,15 \mathrm{mo}$ & $\geq 6 \mathrm{mo}$ \\
\hline Quebec & $\checkmark$ & Grade 4 & $12,18 \mathrm{mo}$ & $\checkmark$ & Grade 9 & $\mathrm{~N} / \mathrm{A}$ & $12 \mathrm{mo}$ & $2,4,12 \mathrm{mo}$ & $6-23 \mathrm{mo}$ \\
\hline New Brunswick & $\checkmark$ & $0,2,6 \mathrm{mo}$ & $12,18 \mathrm{mo}$ & $\checkmark$ & Grade 9 & $12 \mathrm{mo}$ & $12 \mathrm{mo}$ & $2,4,6,18 \mathrm{mo}$ & $6-23 \mathrm{mo}$ \\
\hline Nova Scotia & $\checkmark$ & Grade 4 & $12 \mathrm{mo}, 4-6 \mathrm{yrs}$ & $\checkmark$ & Grade 10 & $12 \mathrm{mo}$ & $12 \mathrm{mo}$ & $2,4,6,18 \mathrm{mo}$ & $6-23 \mathrm{mo}$ \\
\hline PEI & $\checkmark$ & $2,4,15 \mathrm{mo}$ & $15,18 \mathrm{mo}$ & $\checkmark$ & Grade 9 & $12 \mathrm{mo}$ & $12 \mathrm{mo}$ & $2,4,6,18 \mathrm{mo}$ & $\mathrm{N} / \mathrm{A}$ \\
\hline NL & $\checkmark$ & Grade 4 & $12,18 \mathrm{mo}$ & $\checkmark$ & Grade 9 & $12 \mathrm{mo}$ & $12 \mathrm{mo}$ & $2,4,6,18 \mathrm{mo}$ & $\mathrm{N} / \mathrm{A}$ \\
\hline Northwest Territories & $\checkmark$ & $0,1,6 \mathrm{mo}$ & $12,18 \mathrm{mo}$ & $\checkmark$ & Grade 9 & $12 \mathrm{mo}$ & $2,4 \mathrm{mo}$ & $\mathrm{N} / \mathrm{A}$ & $6-23 \mathrm{mo}$ \\
\hline Yukon Territory & $\checkmark$ & $\begin{array}{c}2,4,12 \mathrm{mo} ;<19 \mathrm{yrs} \\
\text { not immunized }\end{array}$ & $12,18 \mathrm{mo}$ & $\checkmark$ & Grade 9 & N/A & $2,6 \mathrm{mo}$ & $2,4,6,12-18 \mathrm{mo}$ & $6-23 \mathrm{mo}$ \\
\hline Nunavut & $\checkmark$ & $0,1,9 \mathrm{mo}$ & $12,18 \mathrm{mo}$ & $\checkmark$ & Grade 9 & $12 \mathrm{mo}$ & N/A & $2,4,6,15 \mathrm{mo}$ & $\geq 6 \mathrm{mo}$ \\
\hline
\end{tabular}

DaPT Diphtheria-acellular pertussis-tetanus; dTap Tetanus-diphtheria acellular pertussis vaccine; Hib Haemophilus influenzae type b vaccine; IPV Inactivated polio vaccine; MMR Measles-mumps-rubella vaccine; mo Age in months; N/A Not available; NL Newfoundland and Labrador; PEI Prince Edward Island; yrs Age in years

Correspondence: Dr Noni MacDonald, Department of Paediatrics, IWK Health Centre, 5840 University Avenue, Halifax, Nova Scotia

B3J 3G9. Telephone 902-470-8799, fax 902-470-7812, e-mail noni.macdonald@dal.ca 


\section{TABLE 2}

Catch-up and high-risk immunization programs for children in Canada, June 2005

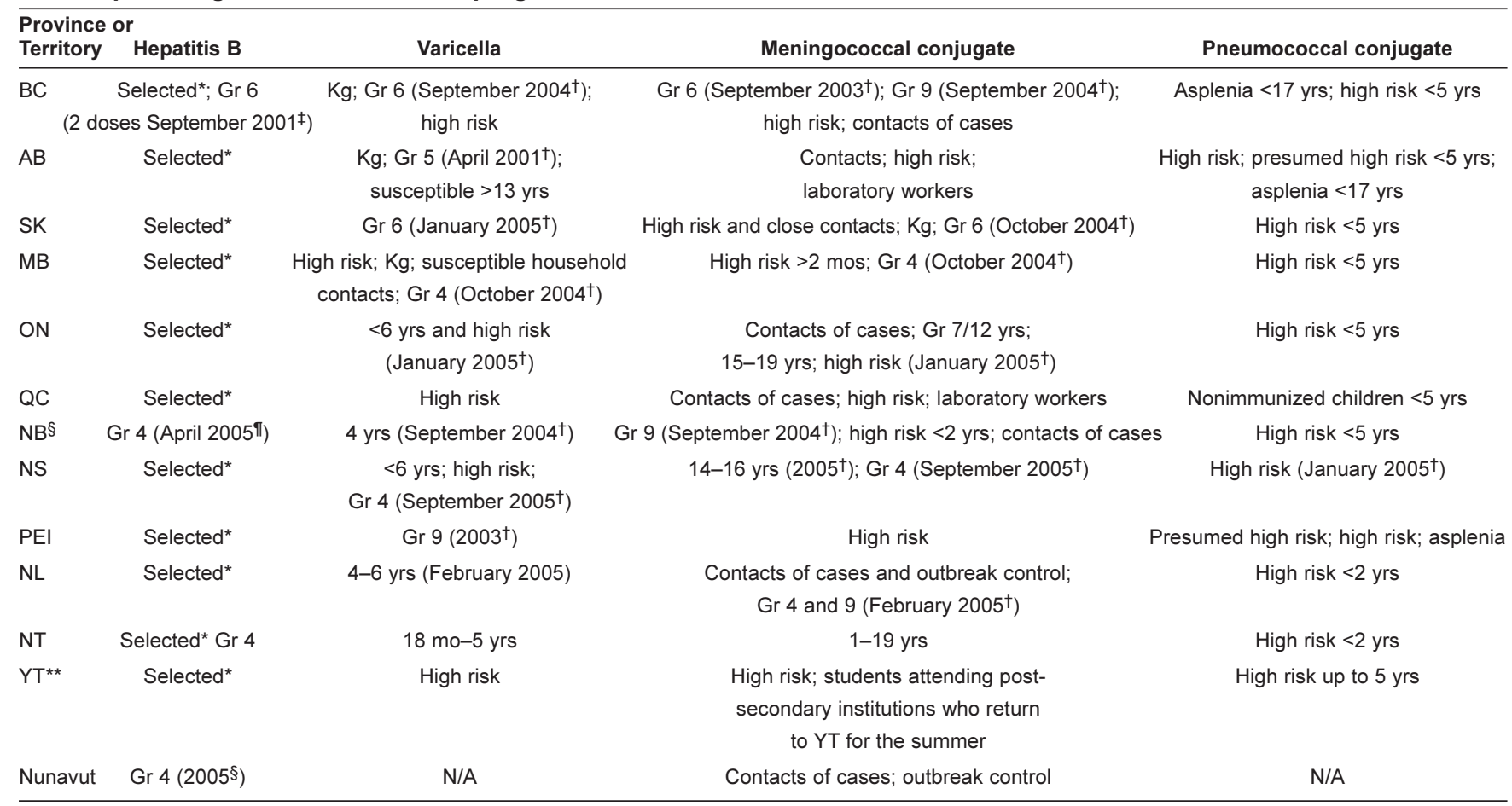

*Prenatal infants, children from endemic countries and/or other selected high-risk groups; †Program began; ¥Two-dose program began; § Tetanus-diphtheria acellular pertussis vaccine (dTap) catch-up for this school year only (2004-2005) for students in grades 10 and 11; ТProgram ends; **dTap catch-up for grade 12 students for 2004-2005 and 2005-2006 only. AB Alberta; BC British Columbia; DaPT Diphtheria-acellular pertussis-tetanus; Gr Grade; Hib Haemophilus influenzae type b vaccine; IPV Inactivated polio vaccine; Kg Kindergarten; MB Manitoba; MMR Measles-mumps-rubella vaccine; mo Age in months; N/A Not available; NB New Brunswick; NL Newfoundland and Labrador; NS Nova Scotia; NT Northwest Territories; ON Ontario; PEI Prince Edward Island; QC Quebec; SK Saskatchewan; yrs Age in years; YT Yukon Territory

\section{REFERENCES}

1. Public Health Agency of Canada. <http://www.phacaspc.gc.ca/im/index.html $>$ (Version current at July 6, 2005).

2. Canadian Paediatric Society, Infectious Diseases and Immunization Committee. Routine Immunization Schedule: Update 2004. Paediatr Child Health 2004;9:17-20.

3. MacDonald NE, Embree J. Access to vaccines: A call to action. Paediatr Child Health 2003;8:11-2.
4. Final Report: National Immunization Strategy. A Report from the F/P/T Advisory Committee on Population Health and Health Security (ACPHHS) to the Conference of F/P/T Deputy Ministers of Health. <http://www.phacaspc.gc.ca/publicat/nat_immunization_03/> (Version current at July 6, 2005).

5. MacDonald NE. Disharmony in provincial and territorial immunization schedules: A downside of recent developments. Paediatr Child Health 1997;2:71-2.

\section{INFECTIOUS DISEASES AND IMMUNIZATION COMMITTEE (2004-2005)}

Members: Drs Upton Allen, The Hospital for Sick Children, Toronto, Ontario; H Dele Davies, Michigan State University, East Lansing, Michigan, USA; Simon Richard Dobson, BC's Children's Hospital, Vancouver, British Columbia; Joanne Embree, The University of Manitoba, Winnipeg, Manitoba (chair); Joanne Langley, IWK Health Centre, Halifax, Nova Scotia; Dorothy Moore, The Montreal Children's Hospital, Montreal, Quebec; Gary Pekeles, The Montreal Children's Hospital, Montreal, Quebec (board representative, 2000-2004); Élisabeth Rousseau-Harsany, Hôpital Sainte-Justine, Montreal, Quebec (board representative).

Consultants: Drs Gilles Delage, Héma-Québec, Saint-Laurent, Quebec; Noni MacDonald, Department of Paediatrics, IWK Health Centre, Halifax, Nova Scotia.

Liaisons: Drs Scott Halperin, IWK Health Centre, Halifax, Nova Scotia (IMPACT); Monica Naus, BC Centre for Disease Control, Vancouver, British Columbia (Health Canada, National Advisory Committee on Immunization); Larry Pickering, Centers for Disease Control and Prevention, Atlanta, Georgia, USA (American Academy of Pediatrics, Committee on Infectious Diseases).

Principal authors: Drs Noni MacDonald, Department of Paediatrics, IWK Health Centre, Halifax, Nova Scotia; Simon Richard Dobson, BC's Children's Hospital, Vancouver, British Columbia; Elaine Sartison, Immunization Program, Disease Control and Prevention Branch, Alberta Health and Wellness, Edmonton, Alberta

The recommendations in this statement do not indicate an exclusive course of treatment or procedure to be followed. Variations, taking into account individual circumstances, may be appropriate. This article has also appeared in a previous issue of Paediatrics $\mathcal{E}$ Child Health (Paediatr Child Health Vol 10 No 6 July/August 2005). 


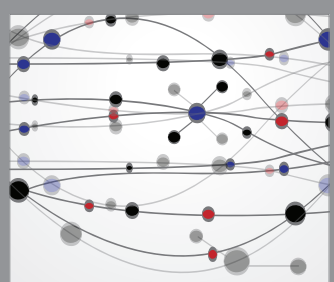

The Scientific World Journal
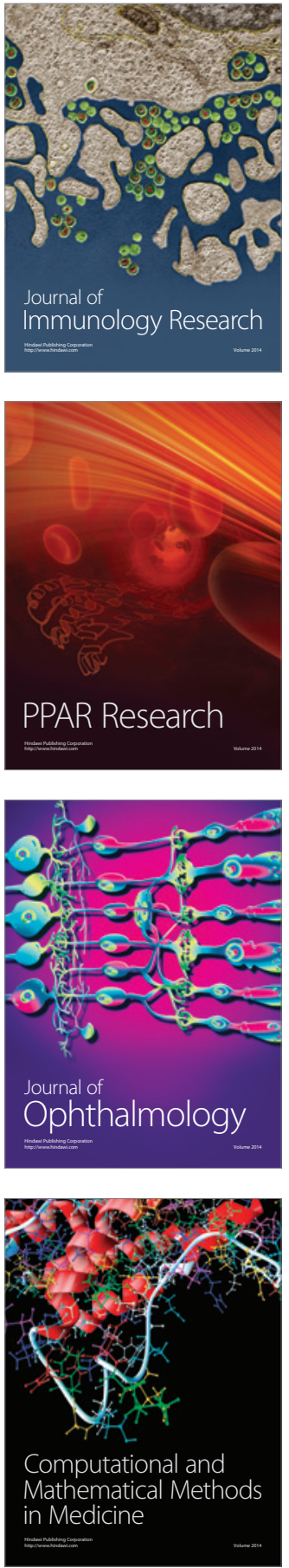

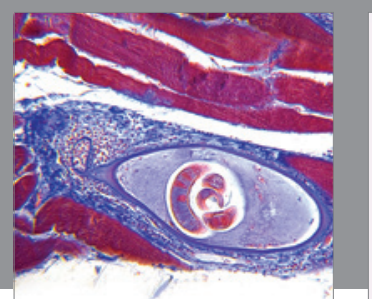

Gastroenterology Research and Practice

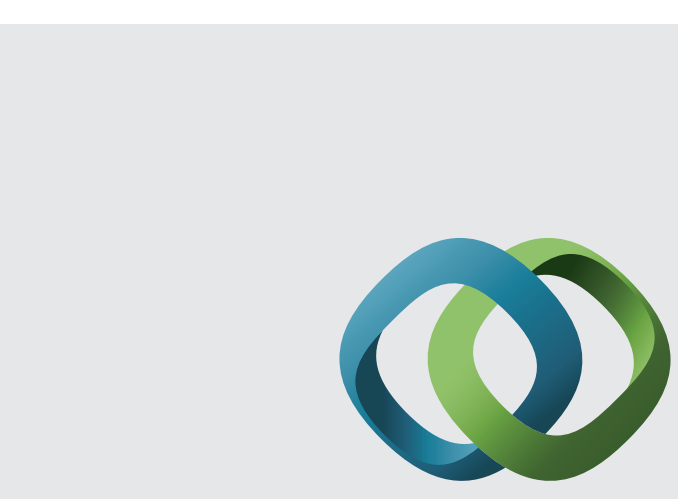

\section{Hindawi}

Submit your manuscripts at

http://www.hindawi.com
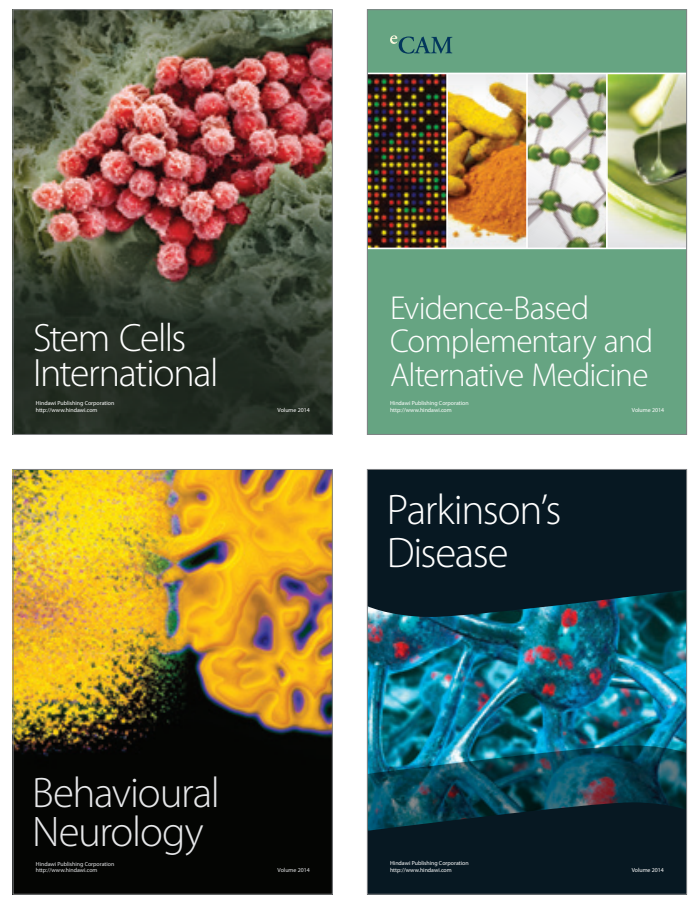
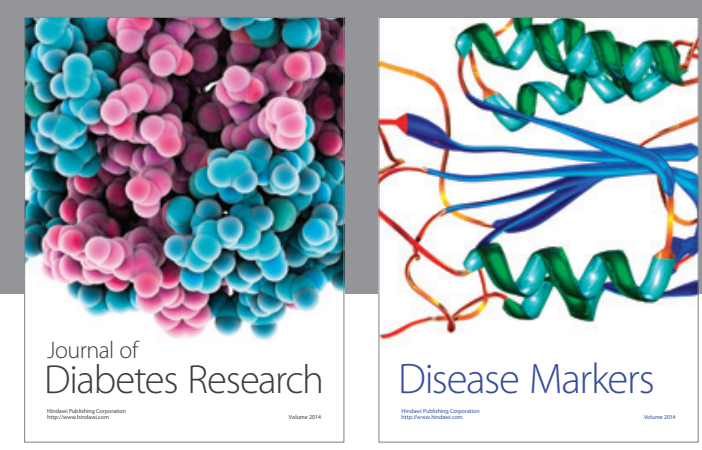

Disease Markers
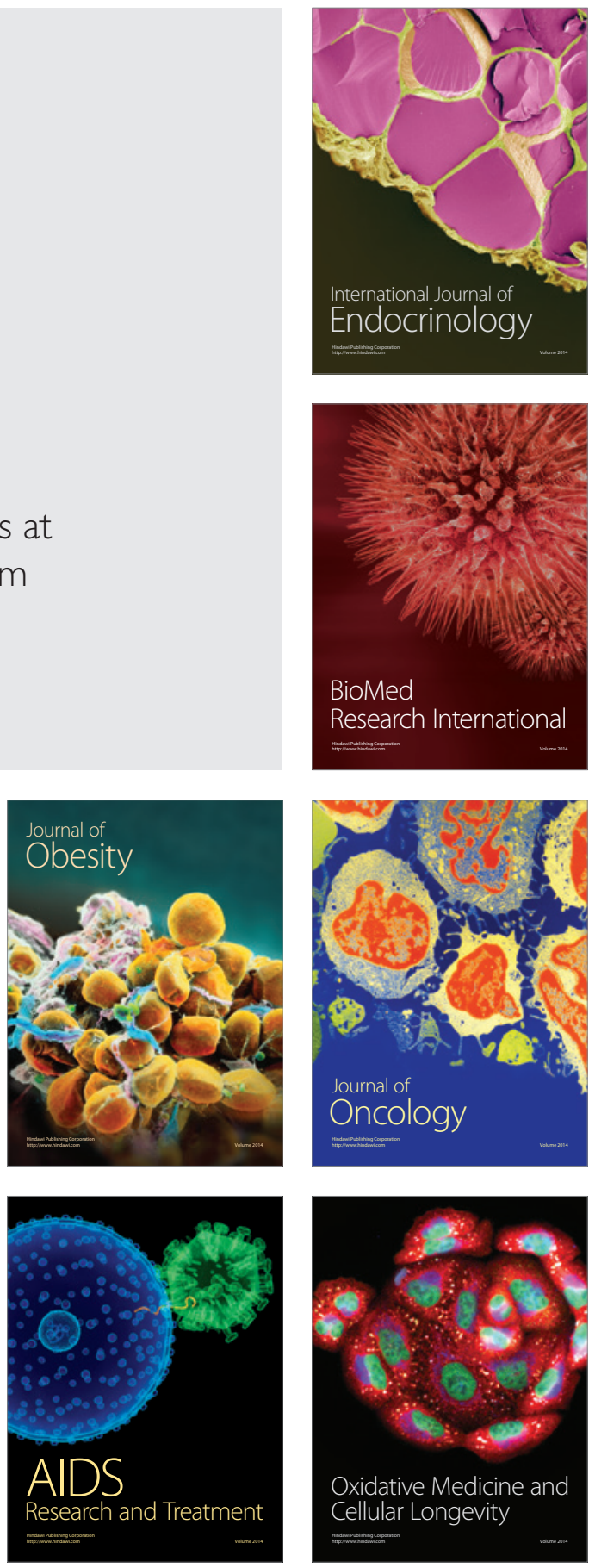